\title{
A Generalized Simple Random Walk in One Dimension Related to the Gaussian Polynomials
}

\section{Taichiro Takagi $\star$}

Department of Physics, Graduate School of Science, University of Tokyo, Hongo, Bunkyo-ku, Tokyo 113, Japan

Received: 1 March 1993/in revised form: 2 August 1993

\begin{abstract}
A generalization of the relation between the simple random walk on a regular lattice and the diffusion equation in a continuous space is described. In one dimension we consider a random walk of a walker with exponentially decreasing mobility with respect to time. It has an exact solution of the conditional probability, that is expressed in terms of the Gaussian polynomials, a generalization of binomial coefficients. Taking a suitable continuum limit we obtain the corresponding transport equation from the recursion relation of the discrete random walk process. The kernel of this differential equation is also directly obtained from that conditional probability by the same continuum limit.
\end{abstract}

\section{Introduction}

It is well known that the diffusion equation in a continuous space is obtained as a continuum limit of the simple random walk process on a regular lattice in arbitrary dimension (see, for example Chapter 1 of [4]). Let us consider the one-dimensional case. The diffusion (or heat) equation

$$
\frac{\partial P}{\partial t}(x, t)=\frac{1}{4} \frac{\partial^{2} P}{\partial x^{2}}(x, t),
$$

has the kernel

$$
P(x, t)=\frac{1}{\sqrt{\pi t}} e^{-x^{2} / t} .
$$

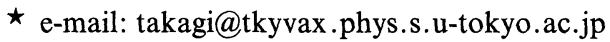

Fellow of the Japan Society for the Promotion of Science for Japanese Junior Scientists 
On the other hand the recursion relation of conditional probabilities of the simple random walk

$$
P[n, m]=\frac{1}{4}(P[n+1, m-1]+2 P[n, m-1]+P[n-1, m-1]),
$$

has the kernel

$$
P[n, m]=\frac{1}{2^{2 m}}\left(\begin{array}{c}
2 m \\
m+n
\end{array}\right),
$$

where $(:)$ is the binomial coefficient. Consider this process to be a random walk only on the even sites of the lattice, namely the probability $P[n, m]$ is associated to the even temporal point $2 m$ and even spatial point $2 n$. The continuous kernel (1.2) can be given from the lattice kernel (1.4) by the following scaling limit. Introducing a small parameter $\varepsilon$, let

$$
x \equiv n \varepsilon, \quad t \equiv m \varepsilon^{2},
$$

be fixed, and let us take the limit,

$$
n \rightarrow \infty, \quad m \rightarrow \infty, \quad \varepsilon \rightarrow 0 .
$$

This means that we will see a long time and large distance behavior of the original discrete process and we have,

$$
\frac{1}{2^{2 m}}\left(\begin{array}{c}
2 m \\
m+n
\end{array}\right) \approx \varepsilon \frac{1}{\sqrt{\pi t}} e^{-x^{2} / t},
$$

where and hereafter $\approx$ means that we have neglected higher order terms with respect to $\varepsilon$ in the right-hand side. The same scaling limit (1.6) leads the recursion relation itself (1.3) to produce the diffusion equation (1.1). To be precise; if $P[n, m]$ is a solution of (1.3) and there exists an analytic function $P(x, t)$ that satisfies $P[n, m]=\varepsilon P(x, t)+o\left(\varepsilon^{2}\right)$ under the scaling limit (1.6), this $P(x, t)$ satisfies the differential equation (1.1). In this sense there is a twofold correspondence between the discrete system and the continuous system.

In one-dimension this correspondence between both kernels can be derived directly in the real space as well as in the momentum space, owing to the above explicit expression of the kernel (1.4) by means of the binomial coefficient. The binomial coefficient appearing in (1.7) has originated from a combinatorial calculation in random walk. In partition theory a generalization of the set of the binomial coefficients has been known as the set of Gaussian polynomials [1], that was originally studied by Gauss about one hundred and thirty years ago. About ten years ago Andrews et al. [2] have used these polynomials in the study of models of statistical mechanics in two-dimensional space, and a generalization of the simple random walk in finite intervals has been presented in their paper (Theorem 2.3.1. of [2]). A natural question arises: can we take a continuum limit of this random walk process as well as we have done above with the usual simple random walk?

In this paper a generalization of the correspondence between discrete random walks and continuous transport phenomena is exposed, keeping in mind the generalization of binomial coefficients to Gaussian polynomials. In Sect. 2 we investigate a random walk process on a lattice of a walker whose mobility decreases with respect to time exponentially, and show that a suitable scaling limit produces a transport phenomenon in a continuous space. The correspondence between the system on a lattice and that in a continuous space is twofold also in 
this case. The recursion relation goes to the differential equation while the solution of the former, that is expressed in terms of the Gaussian polynomial, goes to that of the latter. This is considered in Sect. 3 and we present a proposition (Proposition 2) that is a generalization of (1.7). In Sect. 4 we give a summary. Proofs of the propositions are given in Appendices. Since Proposition 2 is the main result in the present paper, we shall give a full calculation of its proof in Appendix B. The proof itself seems to have some interesting mathematics.

\section{A Generalized Simple Random Walk and its Continuum Limit}

In this section we present a generalization of the simple random walk in one dimension and consider its continuum limit. We introduce correlations between two successive steps in the simple random walk, at the same time we also introduce a time dependence in the reversing probability per unit time. This turns out, as we will see later, to cause a rather simple modification on the heat equation. The process is governed by a set of two coupling recursion relations,

$$
\begin{aligned}
\left(1+q^{m}\right)\left(1+q^{m-1 / 2}\right) P^{ \pm}[n, m ; q]= & P^{ \pm}[n, m-1 ; q] \\
& +q^{m} P^{ \pm}[n, m-1 ; q] \\
& +q^{m-1 / 2} P^{ \pm}[n \pm 1, m-1 ; q] \\
& +q^{2 m-1 / 2} P^{ \pm}[n \mp 1, m-1 ; q],
\end{aligned}
$$

with the initial condition,

$$
P^{ \pm}[n, 0 ; q]=\frac{1}{2} \cdot \delta_{n, 0},
$$

where $0<q<1$. The coefficients such as $q^{m}$, etc. show the walkers' exponentially decreasing mobility with respect to time. As mentioned before notations are accommodated to be in the even sub-lattice. In terms of the original lattice system. $P^{ \pm}[n, m ; q]$ is the probability that the walker is located at $2 n$ at time $2 m$, and at $2 n \pm 1$ at time $2 m+1$. In the step from time $M$ to $M+1$ the walker reverses (returns to the point where he was located at time $M-1)$ with probability $1 /\left(1+q^{M / 2}\right)$ or goes straight with probability $q^{M / 2} /\left(1+q^{M / 2}\right)$. This intermediate process $2 m-1 \rightarrow 2 m+1$ of three successive steps $2 m-2 \rightarrow 2 m-1 \rightarrow 2 m \rightarrow 2 m+1$ has been summed up to give the set of recursion relations (2.1). The conditional probability for time $2 m$ and coordinate $2 n$ is therefore defined as

$$
P[n, m ; q]=P^{+}[n, m ; q]+P^{-}[n, m ; q] .
$$

This process is a $q$-deformation of the simple random walk. If $q$ goes to 1 then (2.1) goes to (1.3). Since this process is linear and spatially translational invariant, finding the fundamental solution (kernel) with this particular initial condition (2.2) is sufficient to construct a Green function for any boundary condition. In the course of the calculation of one-point function for regime III RSOS model in [2], by the so-called corner transfer matrix method, they considered a Dirichlet problem of this process (Theorem 2.3.1. of [2]). That is a model of 2-dimensional statistical mechanics describing a certain series of critical phenomena where $q=0$ corresponds to the low temperature limit and $q=1$ is considered as a multicritical point. We shall display the kernel in the next section. 
We consider the following scaling limit for this process. In addition to (1.6) let $q$ go to 1 . Let.

$$
x \equiv n \varepsilon, \quad t \equiv m \varepsilon^{2}, \quad \lambda \equiv-(\log q) / \varepsilon^{2},
$$

be fixed and take the limit,

$$
n \rightarrow \infty, \quad m \rightarrow \infty, \quad q \rightarrow 1, \quad \varepsilon \rightarrow 0 .
$$

We assume the scaling limit (2.5) of $P^{ \pm}[n, m ; q]$ as

$$
P^{ \pm}[n, m ; q]=\frac{\varepsilon}{2} P(x, t ; \lambda) \pm \varepsilon^{2} Q(x, t ; \lambda)+o\left(\varepsilon^{2}\right) .
$$

Let us substitute them in one of the recursion relations (2.1),

$$
\begin{aligned}
& \left(1+e^{-\lambda t}\right)\left(1+e^{-\lambda t+\lambda \varepsilon^{2} / 2}\right)\left[\frac{1}{2} P(x, t ; \lambda)+\varepsilon Q(x, t ; \lambda)\right] \\
& =\left[\frac{1}{2} P\left(x, t-\varepsilon^{2} ; \lambda\right)+\varepsilon Q\left(x, t-\varepsilon^{2} ; \lambda\right)\right] \\
& \quad+e^{-\lambda t}\left[\frac{1}{2} P\left(x, t-\varepsilon^{2} ; \lambda\right)-\varepsilon Q\left(x, t-\varepsilon^{2} ; \lambda\right)\right] \\
& \quad+e^{-\lambda t+\lambda \varepsilon^{2} / 2}\left[\frac{1}{2} P\left(x+\varepsilon, t-\varepsilon^{2} ; \lambda\right)-\varepsilon Q\left(x+\varepsilon, t-\varepsilon^{2} ; \lambda\right)\right] \\
& \quad+e^{-2 \lambda t+\lambda \varepsilon^{2} / 2}\left[\frac{1}{2} P\left(x-\varepsilon, t-\varepsilon^{2} ; \lambda\right)+\varepsilon Q\left(x-\varepsilon, t-\varepsilon^{2} ; \lambda\right)\right] .
\end{aligned}
$$

The other is just as that with $\varepsilon$ replaced by $-\varepsilon$. Let us expand (2.7) up to $O\left(\varepsilon^{2}\right)$. Due to cancellations of terms in both sides, the $\varepsilon^{2}$ 's in the exponents of the pre-factors have actually no effect on the result. Thus we get,

in $O(\varepsilon)$ and,

$$
Q(x, t ; \lambda)=\frac{1}{8}\left(1-e^{-\lambda t}\right) \frac{\partial P}{\partial x}(x, t ; \lambda)
$$

$$
\frac{1}{2}\left(1+e^{-\lambda t}\right) \frac{\partial P}{\partial t}(x, t ; \lambda)=e^{-\lambda t}\left(\frac{1}{4} \frac{\partial^{2} P}{\partial x^{2}}(x, t ; \lambda)-\frac{\partial Q}{\partial x}(x, t ; \lambda)\right),
$$

in $O\left(\varepsilon^{2}\right)$. Putting them together we have a transport equation,

$$
\frac{\partial P}{\partial t}(x, t ; \lambda)=\frac{e^{-\lambda t}}{4} \frac{\partial^{2} P}{\partial x^{2}}(x, t ; \lambda) .
$$

The coefficient is modified by the factor $e^{-\lambda t}$ in this case, depending on time as it should be. This equation can be easily derived from the heat equation (1.1) in another way. If $P(x, t)$ is a solution of the heat equation $(1.1)$ then $P\left(x,\left(1-e^{-\lambda t}\right) / \lambda\right)$ satisfies Eq. (2.10). From that point of view this generalization of the heat equation is somewhat trivial. However we emphasize here that we have naturally derived this Eq. (2.10) from a corresponding discrete process, this is independent of that transformation on the time variable. We shall investigate this continuum limit in the next section from another point of view. 


\section{Kernel of the Generalized Simple Random Walk and its Continuum Limit}

We have obtained a rather simple transport equation (2.10) as the scaling limit of the recursion relation. In fact the set of solutions of (2.1) with the initial condition (2.2) is explicitly expressed as:

\section{Proposition 1.}

$$
P^{ \pm}[n, m ; q]=\frac{q^{n^{2} \pm n / 2}}{\prod_{k=0}^{2 m}\left(1+q^{(k / 2)}\right)}\left[\begin{array}{c}
2 m \\
m+n
\end{array}\right],
$$

where [:] is a Gaussian polynomial.

A proof is given in Appendix A, that is essentially the same that they have shown in [2]. Only the following two recursion relations of the Gaussian polynomials [1] are needed for the proof,

$$
\begin{aligned}
& {\left[\begin{array}{l}
n \\
m
\end{array}\right]=\left[\begin{array}{c}
n-1 \\
m-1
\end{array}\right]+q^{m}\left[\begin{array}{c}
n-1 \\
m
\end{array}\right],} \\
& {\left[\begin{array}{l}
n \\
m
\end{array}\right]=q^{n-m}\left[\begin{array}{c}
n-1 \\
m-1
\end{array}\right]+\left[\begin{array}{c}
n-1 \\
m
\end{array}\right] .}
\end{aligned}
$$

Both of them are $q$-analogues of the recursion relation of the binomial coefficients. Remarkably they are compatible. Explicitly the Gaussian polynomials are defined by,

$$
\left[\begin{array}{l}
n \\
m
\end{array}\right]= \begin{cases}(q)_{n}(q)_{m}^{-1}(q)_{n-m}^{-1} & \text { if } 0 \leqq m \leqq n \\
0 & \text { otherwise }\end{cases}
$$

where $(q)_{n}=\prod_{k=1}^{n}\left(1-q^{k}\right)$ and $(q)_{0}=1$. Thereby these polynomials satisfy the two recursion relations (3.2) and (3.3). Then the solutions (3.1) also satisfy the initial condition (2.2), and we can see that the conditional probability (2.3) is in fact a $q$-analogue of the kernel of the simple random walk (1.4), that is recovered as $q \rightarrow 1$ of (2.3). Now let us apply the scaling limit (2.5) on the conditional probability (2.3) itself. We present the result as a proposition, that is the main result of the present paper. So far our discussion is also valid for $q>1$, but in the sequel the constraint $0<q<1$ is necessary for the proof of the proposition.

Proposition 2. Let

$$
x \equiv n \varepsilon, \quad t \equiv m \varepsilon^{2}, \quad \lambda \equiv-(\log q) / \varepsilon^{2},
$$

be fixed and take the limit,

$$
n \rightarrow \infty, \quad m \rightarrow \infty, \quad q \rightarrow 1, \quad \varepsilon \rightarrow 0 .
$$

Then

$$
\frac{q^{n^{2}}\left(q^{n / 2}+q^{-n / 2}\right)}{\prod_{k=0}^{2 m}\left(1+q^{(k / 2)}\right)}\left[\begin{array}{c}
2 m \\
m+n
\end{array}\right] \approx \varepsilon \cdot \sqrt{\frac{\lambda}{\pi\left(1-e^{-\lambda t}\right)}} \exp \left[-\frac{\lambda x^{2}}{1-e^{-\lambda t}}\right] .
$$


This proposition is a generalization of (1.7), that is recovered as $\lambda \rightarrow 0$. It means that the assumption (2.6) is indeed correct with

$$
P(x, t ; \lambda)=\sqrt{\frac{\lambda}{\pi\left(1-e^{-\lambda t}\right)}} \exp \left[-\frac{\lambda x^{2}}{1-e^{-\lambda t}}\right] .
$$

According to this probability density the variance $\left\langle x^{2}\right\rangle$ grows linearly for small $t$, and exponentially saturates to $1 / \lambda$. We can see that this $P(x, t ; \lambda)$ satisfies the previously obtained Eq. (2.10) with the initial condition $P(x, 0 ; \lambda)=\delta(x)$, namely it is the fundamental solution of this equation. As we have mentioned in the end of Sect. 2, this solution is related to the ordinary heat kernel as $P(x, t ; \lambda)=$ $P\left(x,\left(1-e^{-\lambda t}\right) / \lambda\right)$, where the right-hand side $P$ stands for the fundamental solution $P(x, t)$ that appeared in (1.2). Although from that point of view this is an expected result, an intriguing fact is that we can prove (3.5) directly, without resorting to the assumption (2.6) and Eq. (2.10). We shall give a proof in Appendix B.

Consequently we have established a twofold correspondence between the discrete process and the continuous process. That is, one is that of the recursion relation (2.1) of the generalized simple random walk and the differential equation (2.10), and the other is that of their fundamental solutions ((3.1) and (3.6)).

\section{Summary}

In this paper we have generalized a well-known correspondence between the simple random walk in one-dimension and the diffusion equation. In another aspect we have generalized the relation between the probability for binomial distribution and the heat kernel. Our main result is Proposition 2, that contains the known result (1.7) as a special case. The generalization of the simple random walk has been done along with the $q$-deformation of the binomial coefficients to the Gaussian polynomials. In addition to the continuum limit of space and time coordinates, we took $q$ to 1 simultaneously. We have succeeded to extract a feature of the Gaussian polynomials and have obtained that simple differential equation (2.10) and its fundamental solution (3.6). It should be emphasized again here that each has been obtained independently from the corresponding result on the lattice, without referring to one another. The result obtained in the limit should describe a long distance and long time behavior of the generalized simple random walk process on a lattice when the system is approaching the usual simple random walk.

\section{Appendices}

\section{A. Proof of Proposition 1}

Proof of Proposition 1. According to the two relations (3.2) and (3.3),

$$
\begin{aligned}
{\left[\begin{array}{c}
2 m \\
m+n
\end{array}\right]=} & q^{m-n}\left[\begin{array}{c}
2 m-1 \\
m+n-1
\end{array}\right]+\left[\begin{array}{c}
2 m-1 \\
m+n
\end{array}\right] \\
= & q^{m-n}\left\{q^{m-n}\left[\begin{array}{c}
2 m-2 \\
m+n-2
\end{array}\right]+\left[\begin{array}{c}
2 m-2 \\
m+n-1
\end{array}\right]\right\} \\
& +\left[\begin{array}{c}
2 m-2 \\
m+n-1
\end{array}\right]+q^{m+n}\left[\begin{array}{c}
2 m-2 \\
m+n
\end{array}\right] .
\end{aligned}
$$


Therefore we have

$$
\begin{aligned}
q^{n^{2}+n / 2}\left[\begin{array}{c}
2 m \\
m+n
\end{array}\right]= & q^{2 m-1 / 2+(n-1)^{2}+(n-1) / 2}\left[\begin{array}{c}
2(m-1) \\
(m-1)+(n-1)
\end{array}\right] \\
& +q^{m+n^{2}-n / 2}\left[\begin{array}{c}
2(m-1) \\
(m-1)+n
\end{array}\right]+q^{n^{2}+n / 2}\left[\begin{array}{c}
2(m-1) \\
(m-1)+n
\end{array}\right] \\
& +q^{m-1 / 2+(n+1)^{2}-(n+1) / 2}\left[\begin{array}{c}
2(m-1) \\
(m-1)+(n+1)
\end{array}\right] .
\end{aligned}
$$

This is equivalent to the upper sign of (2.1) if $P^{ \pm}[n, m ; q] \mathrm{s}$ are so defined as in (3.1). Similarly we have,

$$
\begin{aligned}
{\left[\begin{array}{c}
2 m \\
m+n
\end{array}\right]=} & {\left[\begin{array}{c}
2 m-1 \\
m+n-1
\end{array}\right]+q^{m+n}\left[\begin{array}{c}
2 m-1 \\
m+n
\end{array}\right] } \\
= & q^{m-n}\left[\begin{array}{c}
2 m-2 \\
m+n-2
\end{array}\right]+\left[\begin{array}{c}
2 m-2 \\
m+n-1
\end{array}\right] \\
& +q^{m+n}\left\{\left[\begin{array}{c}
2 m-2 \\
m+n-1
\end{array}\right]+q^{m+n}\left[\begin{array}{c}
2 m-2 \\
m+n
\end{array}\right]\right\},
\end{aligned}
$$

and this proves the lower sign of (2.1) as follows,

$$
\begin{aligned}
q^{n^{2}-n / 2}\left[\begin{array}{c}
2 m \\
m+n
\end{array}\right]= & q^{m-1 / 2+(n-1)^{2}+(n-1) / 2}\left[\begin{array}{c}
2(m-1) \\
(m-1)+(n-1)
\end{array}\right] \\
& +q^{n^{2}-n / 2}\left[\begin{array}{c}
2(m-1) \\
(m-1)+n
\end{array}\right]+q^{m+n^{2}+n / 2}\left[\begin{array}{c}
2(m-1) \\
(m-1)+n
\end{array}\right] \\
& +q^{2 m-1 / 2+(n+1)^{2}-(n+1) / 2}\left[\begin{array}{c}
2(m-1) \\
(m-1)+(n+1)
\end{array}\right] .
\end{aligned}
$$

\section{B. Proof of Proposition 2}

Proof of Proposition 2. Since

$$
\frac{q^{n^{2}}\left(q^{n / 2}+q^{-n / 2}\right)}{2} \approx e^{-\lambda x^{2}}
$$

is obvious, it suffices to prove,

$$
\frac{1}{\prod_{k=1}^{2 m}\left(1+q^{(k / 2)}\right)}\left[\begin{array}{c}
2 m \\
m+n
\end{array}\right] \approx \varepsilon \cdot \sqrt{\frac{\lambda}{\pi\left(1-e^{-\lambda t}\right)}} \exp \left[-\frac{e^{-\lambda t} \lambda x^{2}}{1-e^{-\lambda t}}\right] .
$$


Let us introduce the $q$-gamma function [3]

where

$$
\Gamma_{q}(x)=\frac{(q ; q)_{\infty}}{\left(q^{x} ; q\right)_{\infty}}(1-q)^{1-x}
$$

$$
(a ; q)_{\infty}=\prod_{n=0}^{\infty}\left(1-a q^{n}\right)
$$

The gaussian polynomial factor in (B.2) can be expressed in terms of $q$-gamma functions, $\left[\begin{array}{c}2 m \\ m+n\end{array}\right]=\Gamma_{q}(2 m+1) / \Gamma_{q}(m+n+1) \Gamma_{q}(m-n+1)$. Furthermore the LHS of (B.2) itself can be expressed only by $q$-gamma functions,

$$
\frac{1}{\prod_{k=1}^{2 m}\left(1+q^{(k / 2)}\right)}\left[\begin{array}{c}
2 m \\
m+n
\end{array}\right]=\frac{\Gamma_{q}(m+1) \Gamma_{q}\left(m+\frac{1}{2}\right)}{\Gamma_{q}(m+n+1) \Gamma_{q}(m-n+1) \Gamma_{q}\left(\frac{1}{2}\right)} .
$$

It can be shown as follows;

$$
\begin{aligned}
\frac{1}{\prod_{k=1}^{2 m}\left(1+q^{(k / 2)}\right)} & =\prod_{k=1}^{\infty}\left(\frac{1+q^{(m+k / 2)}}{1+q^{(k / 2)}}\right) \\
& =\frac{\left(q^{2 m+1} ; q\right)_{\infty}}{\left(q^{m+1 / 2} ; q^{1 / 2}\right)_{\infty}} \frac{\left(q^{1 / 2} ; q^{1 / 2}\right)_{\infty}}{(q ; q)_{\infty}} \\
& =\frac{\Gamma_{q^{1 / 2}}(2 m+1)}{\Gamma_{q}(2 m+1)} \frac{\left(1-q^{1 / 2}\right)^{2 m}}{(1-q)^{2 m}}
\end{aligned}
$$

therefore

$$
\begin{aligned}
\frac{\Gamma_{q}(2 m+1)}{\prod_{k=1}^{2 m}\left(1+q^{(k / 2)}\right)}= & \left(1+q^{1 / 2}\right)^{-2 m} \Gamma_{q^{1 / 2}}(2 m+1) \\
= & \left(1+q^{1 / 2}\right)^{-2 m} \frac{1-q^{m}}{1-q^{1 / 2}} \Gamma_{q^{1 / 2}}(2 m) \\
= & \left(1+q^{1 / 2}\right)^{-2 m} \frac{1-q^{m}}{1-q^{1 / 2}} \\
& \times \Gamma_{q}(m) \Gamma_{q}\left(m+\frac{1}{2}\right)\left(1+q^{1 / 2}\right)^{2 m-1} / \Gamma_{q}\left(\frac{1}{2}\right) \\
= & \frac{1-q^{m}}{1-q} \Gamma_{q}(m) \Gamma_{q}\left(m+\frac{1}{2}\right) / \Gamma_{q}\left(\frac{1}{2}\right) \\
= & \Gamma_{q}(m+1) \Gamma_{q}\left(m+\frac{1}{2}\right) / \Gamma_{q}\left(\frac{1}{2}\right) .
\end{aligned}
$$


Here we have used a $q$-analogue of Legendre's duplication formula [3]. Then the scaling limit can be estimated as follows,

$$
\begin{gathered}
\frac{\Gamma_{q}(m+1)^{2}}{\Gamma_{q}(m+n+1) \Gamma_{q}(m-n+1)} \approx \exp \left[-\frac{e^{-\lambda t} \lambda x^{2}}{1-e^{-\lambda t}}\right], \\
\frac{\Gamma_{q}\left(m+\frac{1}{2}\right)}{\Gamma_{q}(m+1)} \approx \varepsilon \cdot \sqrt{\frac{\lambda}{1-e^{-\lambda t}}}, \\
\frac{1}{\Gamma_{q}\left(\frac{1}{2}\right)} \approx \frac{1}{\sqrt{\pi}} .
\end{gathered}
$$

The last estimation (B.10) is trivial, $\Gamma$ of $(1 / 2) \approx \Gamma(1 / 2)=\sqrt{\pi}$. The second relation comes from

$$
\frac{\Gamma_{q}\left(m+\frac{1}{2}\right)}{\Gamma_{q}(m+1)} \cdot \frac{\Gamma_{q}(m)}{\Gamma_{q}\left(m+\frac{1}{2}\right)}=\frac{1-q}{1-q^{m}} \approx \frac{\lambda \varepsilon^{2}}{1-e^{-\lambda t}}
$$

hence

$$
\frac{\Gamma_{q}\left(m+\frac{1}{2}\right)}{\Gamma_{q}(m+1)} \approx \frac{\Gamma_{q}(m)}{\Gamma_{q}\left(m+\frac{1}{2}\right)} \approx \varepsilon \cdot \sqrt{\frac{\lambda}{1-e^{-\lambda t}}} .
$$

In order to show the estimation (B.8) we can use the $q$-analogue of Stirling's formula [5],

$$
\begin{aligned}
\log \Gamma_{q}(z) \sim(z-1 / 2) \log \left(\frac{1-q^{z}}{1-q}\right)+\frac{1}{\log q} \int_{-\log q}^{-z \log q} \frac{u d u}{e^{u}-1} \\
+C_{q}+\sum_{k=1}^{\infty} \frac{B_{2 k}}{(2 k) !}\left(\frac{\log q}{q^{z}-1}\right)^{2 k-1} q^{z} P_{2 k-3}\left(q^{z}\right)
\end{aligned}
$$

where $C_{q}$ is a constant depending on $q, B_{n}$ is the $n^{\text {th }}$ Bernoulli number and $P_{n}(x)$ is a polynomial of degree $n$. Using this formula we can estimate the LHS of (B.8) as follows;

$$
\begin{aligned}
& \log \frac{\Gamma_{q}(m+1)^{2}}{\Gamma_{q}(m+n+1) \Gamma_{q}(m-n+1)} \\
& \approx \log \frac{\Gamma_{q}(m)^{2}}{\Gamma_{q}(m+n) \Gamma_{q}(m-n)} \\
& \approx 2(m-1 / 2) \log \left(\frac{1-q^{m}}{1-q}\right)
\end{aligned}
$$




$$
\begin{aligned}
& -(m+n-1 / 2) \log \left(\frac{1-q^{m+n}}{1-q}\right)-(m-n-1 / 2) \log \left(\frac{1-q^{m-n}}{1-q}\right) \\
& +\frac{1}{\log q}\left(2 \int_{-\log q}^{-m \log q} \frac{u d u}{e^{u}-1}-\int_{-\log q}^{-(m+n) \log q} \frac{u d u}{e^{u}-1}-\int_{-\log q}^{-(m-n) \log q} \frac{u d u}{e^{u}-1}\right) \\
& \approx m \log \left(\frac{\left(1-q^{m}\right)^{2}}{\left(1-q^{m+n}\right)\left(1-q^{m-n}\right)}\right) \\
& -n \log \left(\frac{1-q^{m+n}}{1-q^{m-n}}\right) \\
& -\frac{1}{\log q}\left(\int_{-m \log q}^{-(m+n) \log q} \frac{u d u}{e^{u}-1}+\int_{-m \log q}^{-(m-n) \log q} \frac{u d u}{e^{u}-1}\right) .
\end{aligned}
$$

The last three terms can be estimated separately.

$$
\begin{aligned}
& m \log \left(\frac{\left(1-q^{m}\right)^{2}}{\left(1-q^{m+n}\right)\left(1-q^{m-n}\right)}\right) \approx \frac{t}{\varepsilon^{2}} \log \left(1-\frac{(\lambda \varepsilon x)^{2} e^{-\lambda t}}{\left(1-e^{-\lambda t}\right)^{2}}\right)^{-1} \\
& \approx \frac{(\lambda t)\left(\lambda x^{2}\right) e^{-\lambda t}}{\left(1-e^{-\lambda t}\right)^{2}} \\
& -n \log \left(\frac{1-q^{m+n}}{1-q^{m-n}}\right) \approx-\frac{x}{\varepsilon} \log \left(1+\frac{\lambda \varepsilon x e^{-\lambda t}}{1-e^{-\lambda t}}\right) \\
& +\frac{x}{\varepsilon} \log \left(1-\frac{\lambda \varepsilon x e^{-\lambda t}}{1-e^{-\lambda t}}\right) \\
& \approx-\frac{2 \lambda x^{2} e^{-\lambda t}}{1-e^{-\lambda t}}, \\
& -\frac{1}{\log q}\left(\int_{-m \log q}^{-(m+n) \log q} \frac{u d u}{e^{u}-1}+\int_{-m \log q}^{-(m-n) \log q} \frac{u d u}{e^{u}-1}\right) \\
& =\frac{x}{\varepsilon} \frac{1}{\lambda \varepsilon x}\left(\int_{\lambda t}^{\lambda t+\lambda \varepsilon x} \frac{u d u}{e^{u}-1}+\int_{\lambda t}^{\lambda t-\lambda \varepsilon x} \frac{u d u}{e^{u}-1}\right) \\
& \approx \frac{x}{\varepsilon} \cdot(\lambda \varepsilon x) \cdot\left[\frac{d}{d u}\left(\frac{u}{e^{u}-1}\right)\right]_{u=\lambda t} \\
& =\lambda x^{2}\left(\frac{1}{e^{\lambda t}-1}-\frac{\lambda t e^{\lambda t}}{\left(e^{\lambda t}-1\right)^{2}}\right) \text {. }
\end{aligned}
$$

Therefore (B.15) $+($ B.16) $+($ B.17) gives the required relation (B.8).

Acknowledgements. The author thanks Prof. Miki Wadati and Dr. Tetsuo Deguchi for stimulating discussions. He also thanks Hideaki Ujino and Kazuhisa Inata for their critical reading of the manuscript. This work is partly supported by the Grant-in-Aid for Scientific Research from the Ministry of Education, Science and Culture, Japan No. 05-3002. 


\section{References}

1. Andrews, G.E.: The Theory of Partitions. Massachusetts: Addison-Wesley, 1976

2. Andrews, G.E., Baxter, R.J., Forrester, P.J.: Eight-Vertex SOS Model and Generalized RogersRamanujan-Type Identities. J. Stat. Phys. 35, 193-266 (1984)

3. Askey, R.: The $q$-Gamma and $q$-Beta Functions. Applicable Analysis 8, 125-141 (1978)

4. Itzykson, C., Drouffe, J.M.: Statistical Field Theory. Cambridge: Cambridge University Press, 1989

5. Moak, D.S.: The $q$-analogue of Stirling's Formula. Rocky Mountain J. Math. 14, 403-413 (1984)

Communicated by D. Brydges 
